\section{Journal of Sciences and \\ Engineering}

Vol. 1, $\mathbf{N}^{\circ}$ 1, 2017

\title{
Environmental Impacts associated with solid waste management practices at Alberto Sabogal Sologuren National Hospital, Callao
}

\author{
Luis Hurtado ${ }^{a}$, Jhonny Valverde Flores ${ }^{a, b}$, *
}

aDepartment of Environmental engineering, University Cesar Vallejo - Lima Norte, C.P. 15314, Lima 39, Peru.

${ }^{b}$ Institute of Sciences and Engineering, Centre of Research and Training to the Regional Development (CINCADER). Lima 39, Peru.

ihoval1@yahoo.es

\begin{abstract}
Resumen
Hoy en día se generan gran cantidad de residuos sólidos a nivel mundial, de los cuales solo un $10 \%$ es reutilizado. La cantidad de residuos sólidos reaprovecharle disminuye si es que los residuos tienen algunas características que los hacen peligrosos, como es el caso de los residuos sólidos hospitalarios. A esto se suman los impactos ambientales que los residuos y su manejo inadecuado conlleva. Esta investigación muestra la asociación de las prácticas de manejo de residuos sólidos con los impactos ambientales en el Hospital Nacional Alberto Sabogal Sologuren. Para ello, se tomó en cuenta la segregación, transporte interno de residuos sólidos, la limpieza y desinfección del equipo y la disponibilidad del equipo de protección personal. Para lograrlo se realizó una evaluación de su manejo en, que se llevó a cabo visitando cada uno de los servicios y/o ambientes del Hospital, una caracterización física de los residuos sólidos durante siete días donde se identificaron y cuantificaron los residuos sólidos de todas las áreas y servicios, una entrevista con el personal que labora en el centro hospitalario y una identificación de factores ambientales que son impactados a consecuencia de las prácticas de manejo de residuos.
\end{abstract}

Palabras clave: Residuos sólidos hospitalarios, manejo, impactos ambientales

\begin{abstract}
Today, a large amount of solid waste is generated worldwide, of which only $10 \%$ is reused. The amount of solid waste reused is reduced if the waste has some characteristics that make it hazardous, as is the case of hospital solid waste (HSW). To this are added the environmental impacts that the waste and its inadequate handling entails. This research shows the association of solid waste management practices with environmental impacts in the Alberto Sabogal Sologuren National Hospital. For this, segregation, internal transport of solid waste, cleaning and disinfection of the equipment and the availability of personal protective equipment were taken into account. To achieve this, an evaluation of its management was carried out, which was carried out by visiting each of the services and / or environments of the Hospital, a physical characterization of solid waste for seven days where solid waste was identified and quantified. areas and services, an interview with staff working in the hospital center and an identification of environmental factors that are impacted as a result of waste management practices.
\end{abstract}

Keywords: Hospital solid waste, management, environmental impacts 


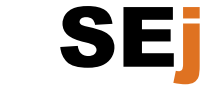

Vol. 1, $\mathbf{N}^{\circ}$ 1, 2017
Journal of Sciences and

Engineering

Copyright @ 2017, CINCADER.

ISSN 2523-9503

\section{Introduction}

Pollution by the waste produced by the human being directly affects our environment and the health of the population. Institutions of different nature seek to reduce the generation of domestic waste and high dangerousness for humans and the environment. This is because there is a lack of knowledge about the management of waste generated in hospitals, clinics, clinics and even more about the impacts caused by them. Many entities in the health sector have realized the importance of proper management of solid waste and are concerned with implementing increasingly effective control measures. In the Alberto Sabogal Sologuren National Hospital, around $88000 \mathrm{~kg} /$ month are generated, causing high costs of external collection and disposal, these are collected by a Service Provider Entity (EPS) and also by the municipality of Bellavista. Among the main problems presented by the Hospital regarding the management of hospital solid waste (HSW) are: lack of equipment and tools for the stages of conditioning, segregation and internal collection, inadequate infrastructure for intermediate storage, accumulated waste in corridors or passages what causes the dissatisfaction of the personnel that works in the center, cross contamination when common waste comes in contact with biocontaminated or special waste, there have been cases of work accidents by sharp objects (hypodermic needles). There are laws that regulate the environmental management of solid waste in Peru. These are: the General Law of the Environment, General Law of Solid Waste, the Technical Health Standard No. 096-MINSA / DIGESA: Management and Management of Solid Waste in Health Establishments and Medical Support Services at a National level.

The modified Leopold matrix uses evaluation criteria assigning scores to the impacts. These are: (1) Direction, (2) Geographical extension, (3) Duration, (4) Magnitude, (5) Frequency, (6) Probability of occurrence; and (7) Reversibility.

Classification of Hospital Waste

According to the Technical Health Standard $N^{\circ}$ 096-MINSA / DIGESA, hospital waste is classified as:
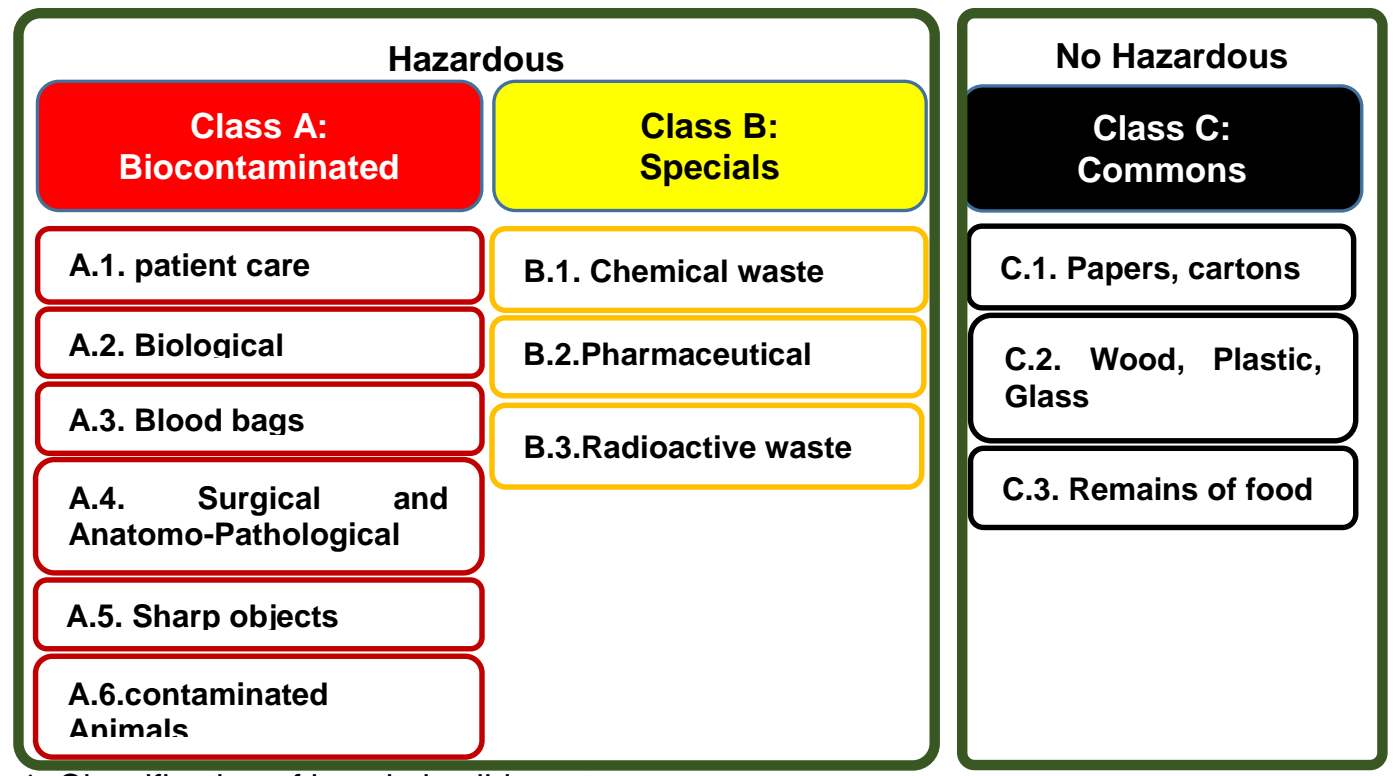

Figure 1. Classification of hospital solid waste

Source: Adapted from Technical Health Standard, № 096-MINSA/DIGESA, 2012 


\section{SEj}

Vol. 1, $\mathrm{N}^{\circ}$ 1, 2017

\section{Journal of Sciences and Engineering}

\section{GINGADER}

Centre of Research and Training for Regional Development Online at www.journals.cincader.org

Stages of the management of Hospital Solid Wastes (HSW) It is important to identify each of the stages of the management of HSW appropriate techniques should be considered in a series of steps that begins with the conditioning materials, supplies and equipment needed to segregate properly, at this stage the waste will be classified according to their nature and danger to avoid cross contamination or other inconveniences, for this reason it is necessary the commitment and participation of the staff of the hospital center. The stages of the management of hospital solid waste are detailed below:
1. Conditioning
2. Primary storage and segregation
3. Intermediate storage
4. Internal collection
5. Internal transport
6. Central storage
7. Treatment (Sterilization by autoclave, Incineration, Microwave disinfection)
8. Collection and transportation outside the hospital facilities
9. Final provision

\section{Materials and Methods}

The present investigation is of non-experimental design since there is no manipulation of any variable. It is correlational in nature, because it studies the relationship between both variables.

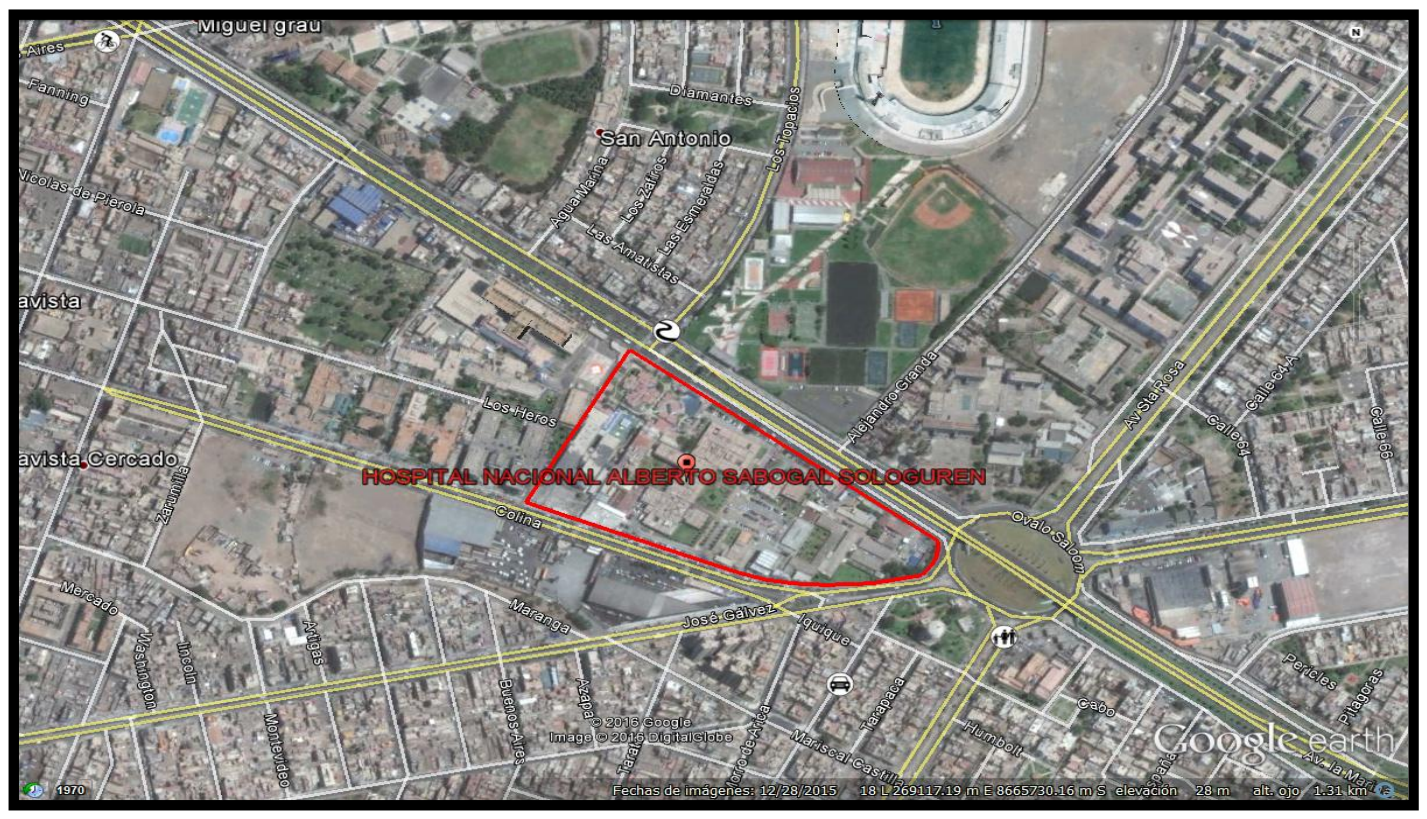

Figure 2. Area of Hospital Nacional Alberto Sabogal Sologuren, Lima, Peru. 


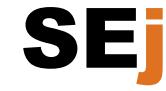

Vol. 1, $\mathrm{N}^{\circ}$ 1, 2017

\section{Journal of Sciences and Engineering}

A publication of

Centre of Research and Training for Regional Development Online at www.journals.cincader.org

The research was carried out at the Alberto Sabogal Sologuren National Hospital for seven days (from May 06 to May 13, 2016). This hospital is located in the district of Bellavista, Province of Callao, Department of Lima, Peru.

Stages of the investigation:

- Identification and quantification of hospital solid waste.

- Evaluation of the management of HSW.

- Determination of environmental impacts.

The modified Leopold matrix with its respective evaluation criteria was used.

Table 1. Evaluation Criteria of the Modified Leopold Matrix.

\begin{tabular}{|c|c|c|}
\hline Criterion & Evaluation & Definition \\
\hline & Positive (+) & Benefit for the resource \\
\hline \multirow[t]{2}{*}{ Direction } & Neutral (0) & No benefit or harm to the resource \\
\hline & Negative (-) & Damage to the resource \\
\hline & Local (1) & Confined to the area directly disturbed by the project \\
\hline \multirow[t]{2}{*}{$\begin{array}{l}\text { Geographical } \\
\text { extension }\end{array}$} & Sub-regional (2) & $\begin{array}{l}\text { It surpasses the areas but it is within the limits of the study area } \\
\text { of the evaluation }\end{array}$ \\
\hline & Regional (3) & It extends beyond the regional limits \\
\hline \multirow[t]{3}{*}{ Duration } & Short term (1) & Less than 1 year \\
\hline & Medium term (2) & Between 1 and 5 years \\
\hline & Long term (3) & More than 5 years \\
\hline \multirow{5}{*}{ Magnitude } & None $(0)$ & No change is expected \\
\hline & $\operatorname{Low}(1)$ & $\begin{array}{l}\text { It is predicted that the disturbance will be somewhat greater } \\
\text { than the typical conditions }\end{array}$ \\
\hline & Medium (2) & $\begin{array}{l}\text { The effects are predicted to be considerably above typical } \\
\text { existing conditions, but without exceeding the criteria }\end{array}$ \\
\hline & & $\begin{array}{l}\text { established in the permissible limits or causing changes in the } \\
\text { economic, social, and biological parameters under the ranges } \\
\text { of natural variability or social tolerance. }\end{array}$ \\
\hline & High (3) & $\begin{array}{l}\text { Predictable effects exceed established criteria or permitted } \\
\text { limits associated with potential adverse effects or cause a } \\
\text { detectable change in economic, social, biological parameters, } \\
\text { beyond natural variability or social tolerance }\end{array}$ \\
\hline \multirow{5}{*}{ Frequency } & Continuous (4) & It will happen continuously \\
\hline & Isolated(3) & Confined to a specific period \\
\hline & Periodical (2) & Occurs intermittently but repeatedly \\
\hline & Occasional (1) & Occurs intermittently and sporadically \\
\hline & Accidental $(0)$ & It happens rarely \\
\hline \multirow{3}{*}{$\begin{array}{l}\text { Probability of } \\
\text { occurrence }\end{array}$} & Low $(0.1-0.3)$ & Unlikely \\
\hline & $\begin{array}{l}\text { Medium } \quad(0.4 \quad- \\
0.7)\end{array}$ & Possible probable \\
\hline & High $(0.8-1.0)$ & Certain \\
\hline \multirow{4}{*}{ Reversibility } & Short term $(0)$ & It can be reversed in a year or less \\
\hline & Medium term (1) & It can be reversed in more than a year, but less than ten. \\
\hline & Long term (2) & It can be reversed in more than ten years \\
\hline & Irreversible (3) & Permanent effects \\
\hline
\end{tabular}




\section{SEj}

Vol. 1, $\mathrm{N}^{\circ}$ 1, 2017
Journal of Sciences and

Engineering

Copyright @ 2017, CINCADER.

ISSN 2523-9503

Table 2. Evaluation criteria for the stages of hospital solid waste management

\begin{tabular}{cl}
\hline Assessment & \multicolumn{1}{c}{ Description } \\
\hline 1 & comply \\
0.5 & Partially Comply \\
0 & Not comply \\
$\mathrm{X}$ & Do not apply \\
\hline
\end{tabular}

Source: Technical Health Standard, Nº 096-MINSA/DIGESA, 2012

Table 3. Criteria for valuation of Checklist N3

\begin{tabular}{cccc}
\hline Very poor & Poor & Acceptable & Satisfied \\
\hline $\begin{array}{c}\text { Score less than } \\
3.5\end{array}$ & $\begin{array}{c}\text { Score between } \\
3.5 \text { and } 5\end{array}$ & $\begin{array}{c}\text { Score equal to or } \\
\text { greater than } 5.5 \text { to } \\
\text { less than } 10\end{array}$ & Score of 10 \\
& & & \\
\hline
\end{tabular}

Source: Technical Health Standard, № 096-MINSA/DIGESA, 2012

Table 4. Value range of importance of impacts

\begin{tabular}{|c|c|c|c|c|}
\hline \multicolumn{3}{|c|}{ Valoración } & Categorización de Impacto & Código de color \\
\hline 15 & $a$ & 10.1 & Altamente positivo & \\
\hline 10 & a & 5.1 & Moderadamente positivo & \\
\hline 5 & a & 0 & Levemente positivo & \\
\hline-0.1 & a & -5 & Levemente negativo & \\
\hline-5.1 & a & -10 & Moderadamente negativo & \\
\hline-10.1 & a & -15 & Altamente negativo & \\
\hline
\end{tabular}

Source: Technical Health Standard, № 096-MINSA/DIGESA, 2012

\section{Results}

\section{Identification and Quantification of Hospital Solid Wastes}

Hospital Solid Waste was identified and quantified. The bags of solid waste generated in each service were identified, these were collected through internal collection to the point of collection, which was the place chosen for the operation, where they proceeded to inspect the inside of each bag. First of all, the waste bags were weighed at the collection point, then the waste volume was determined with the help of a container of known volume, the waste was introduced into the container, ensuring that no empty spaces remained. . When the volume and weight data of the waste were obtained, the waste was classified, the garbage was separated manually according to its type and they were weighed again. Subsequently, the weights of each component were added together and a comparison was made with the total weight of the waste generated. In this way, the percentage by weight of each type of waste was calculated. 
SE

Vol. 1, No 1, 2017
Journal of Sciences and

Engineering

Copyright @ 2017, CINCADER.

ISSN 2523-9503

A publication of

CINCADER

Centre of Research and Training for

Regional Development

Online at www.journals.cincader.org

Table 5. Generation of solid waste by type in each area of the Alberto Sabogal Sologuren National Hospital.

\begin{tabular}{|c|c|c|c|c|c|}
\hline Area & $\begin{array}{l}\text { Biocontaminated } \\
(\mathrm{kg})\end{array}$ & $\begin{array}{l}\text { Special } \\
(\mathrm{kg})\end{array}$ & $\begin{array}{c}\text { Common } \\
(\mathrm{kg})\end{array}$ & Total & $\begin{array}{c}\mathbf{k g} \\
\text { residue/Day }\end{array}$ \\
\hline Medicine I Hospitalization & 620.79 & 0.00 & 157.95 & 778.74 & 111.25 \\
\hline Medicine II Hospitalization & 863.26 & 0.00 & 184.45 & 1047.71 & 149.67 \\
\hline Hemodialysis & 546.04 & 0.00 & 58.05 & 604.09 & 86.30 \\
\hline UCEMI & 653.32 & 0.00 & 125.79 & 779.11 & 111.30 \\
\hline Operations room & 723.23 & 0.00 & 259.59 & 982.82 & 140.40 \\
\hline Cardiology Hospitalization & 327.47 & 0.00 & 107.00 & 434.47 & 62.07 \\
\hline Surgery Hospitalization & 358.50 & 0.00 & 91.86 & 450.36 & 64.34 \\
\hline Urology Hospitalization & 540.17 & 0.00 & 124.64 & 664.81 & 94.97 \\
\hline UCIN & 648.95 & 0.00 & 129.39 & 778.34 & 111.19 \\
\hline Doctor's Office & 279.80 & 0.00 & 262.95 & 542.75 & 77.54 \\
\hline Laboratory & 294.43 & 0.00 & 83.48 & 377.91 & 53.99 \\
\hline Emergency & 1058.22 & 0.00 & 312.05 & 1370.27 & 195.75 \\
\hline Unit Chemoteraphy & 295.70 & 160.58 & 82.04 & 538.32 & 76.90 \\
\hline Medical specialties Hospitalization & 532.78 & 95.06 & 145.03 & 772.87 & 110.41 \\
\hline Pediatric surgery Hospitalization & 422.84 & 0.00 & 135.04 & 557.88 & 79.70 \\
\hline Transitory Hospitalization & 420.75 & 0.00 & 101.76 & 522.51 & 74.64 \\
\hline Blood bank & 478.48 & 0.00 & 114.40 & 592.88 & 84.70 \\
\hline Drugstore & 0.00 & 0.00 & 49.68 & 49.68 & 7.10 \\
\hline Nutrition & 2269.40 & 0.00 & 0.00 & 2269.40 & 324.20 \\
\hline High obstetric Risk & 432.55 & 0.00 & 89.20 & 521.75 & 74.54 \\
\hline Maternity & 666.13 & 0.00 & 52.65 & 718.78 & 102.68 \\
\hline Neonatology & 774.93 & 0.00 & 133.18 & 908.11 & 129.73 \\
\hline Pediatrics Hospitalization & 558.03 & 22.24 & 157.20 & 737.47 & 105.35 \\
\hline Traumatology Hospitalization & 653.13 & 0.00 & 78.67 & 731.80 & 104.54 \\
\hline Neurosurgery Hospitalization & 454.45 & 0.00 & 106.88 & 561.33 & 80.19 \\
\hline $\mathrm{UCl}$ & 735.33 & 0.00 & 133.28 & 868.61 & 124.09 \\
\hline Others & 146.02 & 0.00 & 314.72 & 460.74 & 65.82 \\
\hline Administrative zone & 73.61 & 0.00 & 153.42 & 227.03 & 32.43 \\
\hline Residency & 117.32 & 0.00 & 0.00 & 117.32 & 16.76 \\
\hline Laundry & 0.00 & 0.00 & 246.77 & 246.77 & 35.25 \\
\hline Cartons & 0.00 & 0.00 & 365.67 & 365.67 & 52.24 \\
\hline Total & 15945.63 & 277.88 & 4356.79 & 20580.30 & 2940.04 \\
\hline
\end{tabular}

Source: HSW Characterization of 05/06/2016 - 05/13/2016 


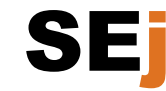

Vol. 1, $\mathrm{N}^{\circ}$ 1, 2017
Journal of Sciences and

Engineering

Copyright (C 2017, CINCADER.

ISSN 2523-9503

A publication of

\section{CINCADER}

Centre of Research and Training for

Regional Development

Online at www.journals.cincader.org

The areas that generate the highest amounts in $\mathrm{kg}$ of biocontaminated waste are nutrition $(2269.40 \mathrm{~kg} /$ week) and emergency $(1058.22 \mathrm{~kg} /$ week). The areas that generate the highest quantities in $\mathrm{kg}$ of special waste are Chemotherapy Unit (160.58 kg / week) and Medical hospitalization specialties (95.06 kg / week). The areas that generate the highest amounts in $\mathrm{kg}$ of common waste are nutrition (324.20 kg / week) and emergency (195.75 kg / week).

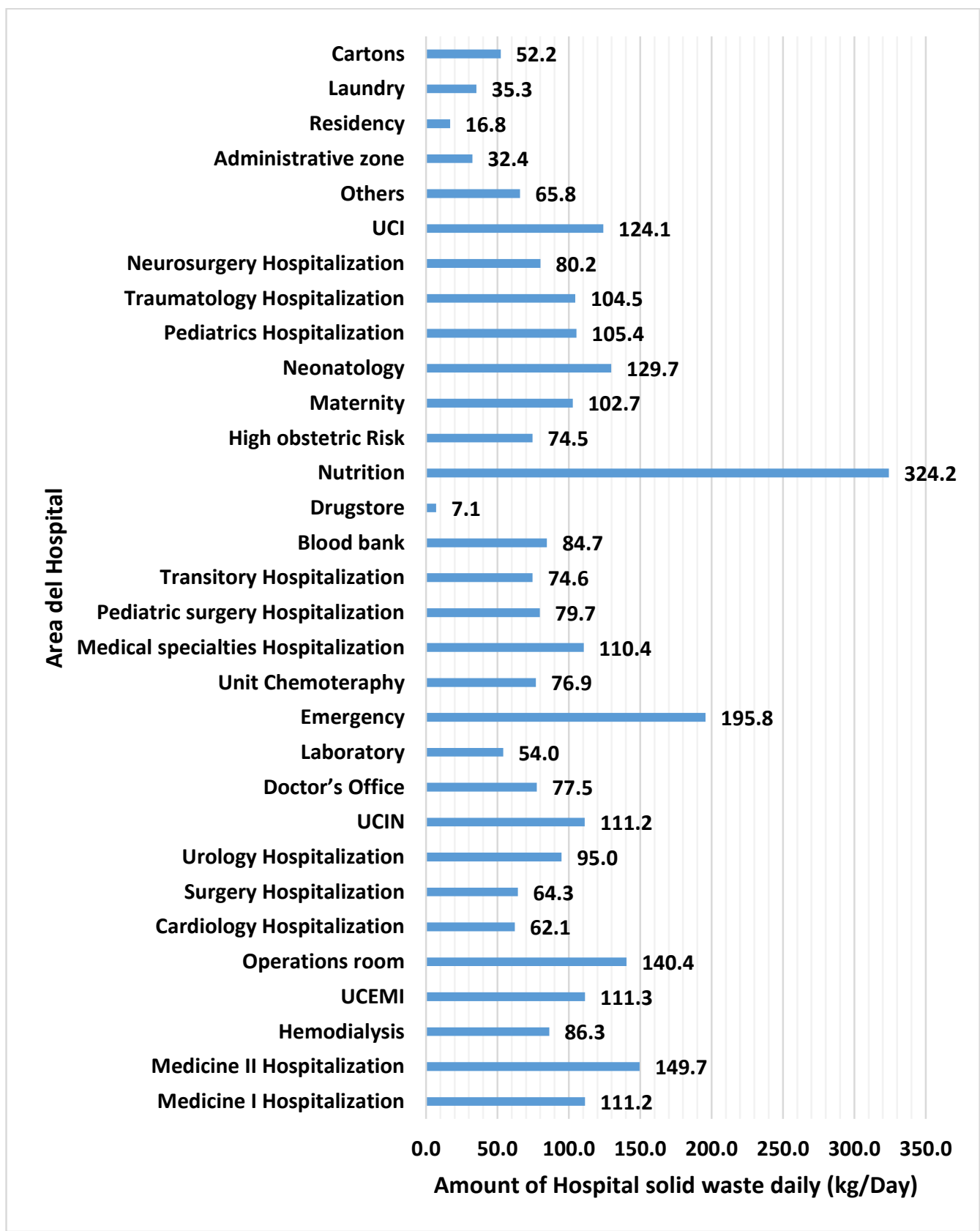

Figure 3. Average hospital solid waste generation in kilograms

Source: HSW Characterization of 05/06/2016 - 05/13/2016 


\section{SEj}

Vol. 1, $\mathrm{N}^{\circ}$ 1, 2017
Journal of Sciences and

Engineering

Copyright @ 2017, CINCADER.

ISSN 2523-9503

The areas that generate the highest quantities in $\mathrm{kg}$ of hospital waste are: nutrition (324.2 $\mathrm{kg} /$ day), emergency (195.8 kg / day), Medicine II Hospitalization (149.7 kg / day), Operating room $(140.4 \mathrm{~kg} /$ day). The areas that generate lower amounts in $\mathrm{kg}$ of hospital waste are: Drugstore ( $7.1 \mathrm{~kg} /$ day), Residency (16.8 kg / day), Administrative zone (32.4 kg / day), and Laundry (35.3 $\mathrm{kg} /$ day).

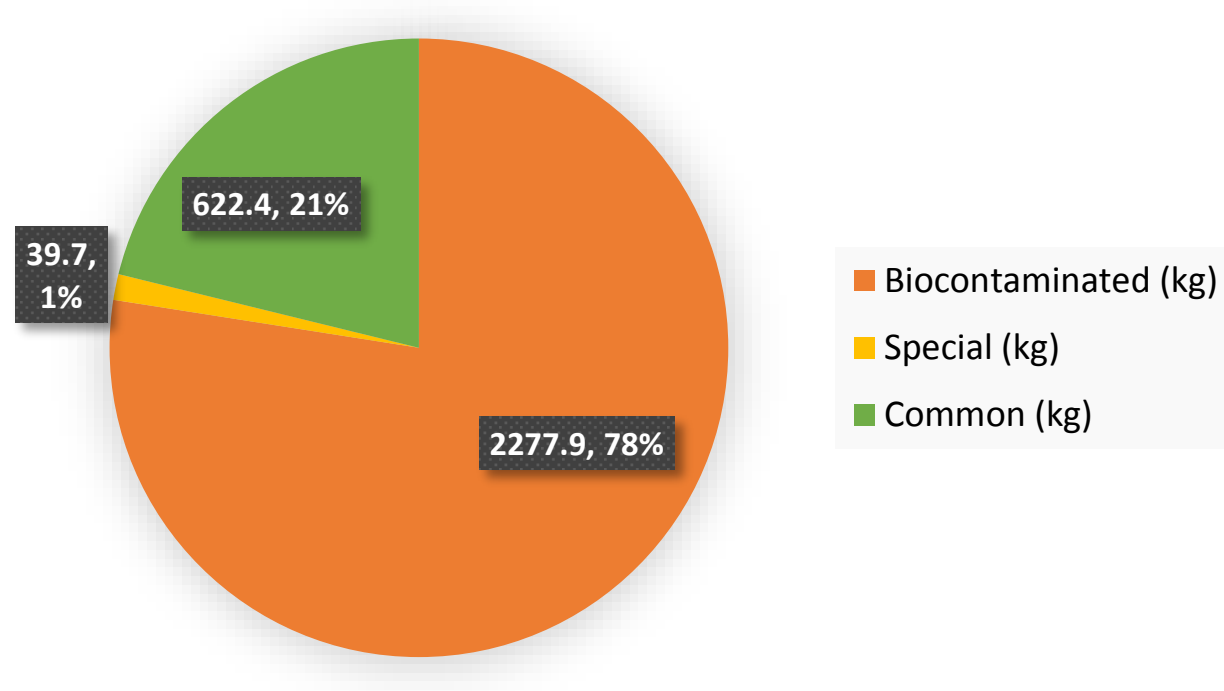

Figure 4. Percentage in Weight by type of waste of the National Hospital Alberto Sabogal Sologuren.

Source: Characterization of HSW of 05/06/2016 - 05/13/2016

Figure 4 represents the percentage by weight of average hospital solid waste generated in the Alberto Sabogal Sologuren National Hospital with each type. Approximately $2940.04 \mathrm{~kg} /$ day of solid waste are generated in total, of which $622.4 \mathrm{~kg}$ are common waste which is equivalent to $21 \%$ of the total generated. The biocontaminated waste reaches up to $2277.9 \mathrm{~kg}$ of generation per day, which represents $78 \%$, while only on average $39.7 \mathrm{~kg}$ of special waste is generated per day, equivalent to $1 \%$ of the total generated.

\section{Evaluation of the management of Hospital Solid Waste}

Table 6. Assessment of the handling of solid waste Hospital 
SE

Vol. 1, $\mathrm{N}^{\circ}$ 1, 2017

Copyright @ 2017, CINCADER.

ISSN 2523-9503
Journal of Sciences and

Engineering

Centre of Research and Training for

Regional Development Online at www.journals.cincader.org

\begin{tabular}{|c|c|c|c|c|c|c|}
\hline Area & Month & 1. Conditioning & $\begin{array}{l}2 . \\
\text { Segregation } \\
\text { and primary } \\
\text { storage }\end{array}$ & $\begin{array}{c}3 . \\
\text { Intermediate } \\
\text { storage }\end{array}$ & Total & Assessment \\
\hline \multirow{3}{*}{$\begin{array}{l}\text { Pediatric surgery } \\
\text { Hospitalization }\end{array}$} & April & 2 & 1.5 & 0 & 3.5 & Deficient \\
\hline & May & 2 & 1.5 & 0 & 3.5 & Deficient \\
\hline & June & 2 & 2 & 0 & 4 & Deficient \\
\hline \multirow{4}{*}{$\begin{array}{c}\text { Transitory } \\
\text { Hospitalization }\end{array}$} & April & 2 & 1.5 & 0 & 3.5 & Deficient \\
\hline & May & 2 & 1.5 & 0 & 3.5 & Deficient \\
\hline & June & 1.5 & 2 & 0 & 3.5 & Deficient \\
\hline & April & 1.5 & 2 & 0 & 3.5 & Deficient \\
\hline \multirow[t]{3}{*}{ Blood bank } & May & 2 & 2 & 0 & 4 & Deficient \\
\hline & June & 2 & 2 & 0 & 4 & Deficient \\
\hline & April & 2 & 1.5 & 0.5 & 4 & Deficient \\
\hline \multirow[t]{3}{*}{ High obstetric Risk } & May & 2 & 1.5 & 0.5 & 4 & Deficient \\
\hline & June & 2 & 1.5 & 1 & 4.5 & Deficient \\
\hline & April & 2 & 1.5 & 0.5 & 4 & Deficient \\
\hline \multirow[t]{3}{*}{ Maternity } & May & 2 & 1.5 & 0.5 & 4 & Deficient \\
\hline & June & 2 & 1.5 & 1 & 4.5 & Deficient \\
\hline & April & 2 & 2 & 0.5 & 4.5 & Deficient \\
\hline \multirow[t]{2}{*}{ Neonatology } & May & 2 & 2 & 0.5 & 4.5 & Deficient \\
\hline & June & 1.5 & 2 & 1 & 4.5 & Deficient \\
\hline \multirow{3}{*}{$\begin{array}{c}\text { Pediatrics } \\
\text { Hospitalization }\end{array}$} & April & 1.5 & 2 & 0 & 3.5 & Deficient \\
\hline & May & 2 & 2 & 0 & 4 & Deficient \\
\hline & June & 2 & 1.5 & 0 & 3.5 & Deficient \\
\hline \multirow{3}{*}{$\begin{array}{l}\text { Traumatology } \\
\text { Hospitalization }\end{array}$} & April & 2 & 2 & 0 & 4 & Deficient \\
\hline & May & 2 & 1.5 & 0 & 3.5 & Deficient \\
\hline & June & 1.5 & 2 & 0 & 3.5 & Deficient \\
\hline \multirow{3}{*}{$\begin{array}{l}\text { Neurosurgery } \\
\text { Hospitalization }\end{array}$} & April & 1.5 & 2 & 0 & 3.5 & Deficient \\
\hline & May & 2 & 2 & 0 & 4 & Deficient \\
\hline & June & 2 & 1.5 & 0 & 3.5 & Deficient \\
\hline \multirow{3}{*}{$\mathrm{UCl}$} & April & 1.5 & 1.5 & 0 & 3 & Very Deficient \\
\hline & May & 2 & 1.5 & 0 & 3.5 & Deficient \\
\hline & June & 2 & 2 & 0 & 4 & Deficient \\
\hline \multirow{3}{*}{ Unit Chemoteraphy } & April & 2 & 2 & 0 & 4 & Deficient \\
\hline & May & 2 & 2 & 0 & 4 & Deficient \\
\hline & June & 2 & 2 & 0 & 4 & Deficient \\
\hline \multirow{3}{*}{$\begin{array}{c}\text { Medical specialties } \\
\text { Hospitalization }\end{array}$} & April & 2 & 1.5 & 0 & 3.5 & Deficient \\
\hline & May & 2 & 2 & 0 & 4 & Deficient \\
\hline & June & 2 & 1.5 & 0 & 3.5 & Deficient \\
\hline
\end{tabular}


SE

Vol. 1, N 1, 2017

Copyright @ 2017, CINCADER.

ISSN 2523-9503
Journal of Sciences and

Engineering

Centre of Research and Training for

Regional Development

Online at www.journals.cincader.org

\begin{tabular}{|c|c|c|c|c|c|c|}
\hline Area & Month & 1. Conditioning & $\begin{array}{l}\text { 2. Segregation } \\
\text { and primary } \\
\text { storage }\end{array}$ & $\begin{array}{c}3 . \\
\text { Intermediate } \\
\text { storage }\end{array}$ & Total & Assessment \\
\hline \multirow{3}{*}{$\begin{array}{c}\text { Medicine II } \\
\text { Hospitalization }\end{array}$} & April & 1.5 & 1.5 & 0 & 3 & Very Deficient \\
\hline & May & 2 & 1.5 & 0 & 3.5 & Deficient \\
\hline & June & 2 & 2 & 0 & 4 & Deficient \\
\hline \multirow{3}{*}{$\begin{array}{c}\text { Medicine I } \\
\text { Hospitalization }\end{array}$} & April & 2 & 1.5 & 0 & 3.5 & Deficient \\
\hline & May & 2 & 2 & 0 & 4 & Deficient \\
\hline & June & 2 & 1.5 & 0 & 3.5 & Deficient \\
\hline \multirow{3}{*}{ Hemodialysis } & April & 2 & 1.5 & 0 & 3.5 & Deficient \\
\hline & May & 2 & 2 & 0 & 4 & Deficient \\
\hline & June & 2 & 2 & 0 & 4 & Deficient \\
\hline \multirow{3}{*}{ UCEMI } & April & 2 & 1.5 & 0 & 3.5 & Deficient \\
\hline & May & 2 & 1.5 & 0 & 3.5 & Deficient \\
\hline & June & 2 & 2 & 0 & 4 & Deficient \\
\hline \multirow{3}{*}{ Operations room } & April & 2 & 2 & 1.5 & 5.5 & Acceptable \\
\hline & May & 2 & 2 & 1.5 & 5.5 & Acceptable \\
\hline & June & 1.5 & 2 & 1.5 & 5 & Deficient \\
\hline \multirow{4}{*}{$\begin{array}{l}\text { Cardiology } \\
\text { Hospitalization }\end{array}$} & April & 2 & 1.5 & 0 & 3.5 & Deficient \\
\hline & May & 2 & 1.5 & 0 & 3.5 & Deficient \\
\hline & June & 2 & 1.5 & 0 & 3.5 & Deficient \\
\hline & April & 1.5 & 1.5 & 0 & 3 & Very Deficient \\
\hline \multirow[t]{3}{*}{ Surgery Hospitalization } & May & 1.5 & 2 & 0 & 3.5 & Deficient \\
\hline & June & 1.5 & 1.5 & 0 & 3 & Very Deficient \\
\hline & April & 2 & 1.5 & 0 & 3.5 & Deficient \\
\hline \multirow[t]{3}{*}{ Urology Hospitalization } & May & 2 & 1.5 & 0 & 3.5 & Deficient \\
\hline & June & 2 & 1.5 & 0 & 3.5 & Deficient \\
\hline & April & 2 & 1.5 & 0 & 3.5 & Deficient \\
\hline \multirow[t]{3}{*}{ UCIN } & May & 2 & 2 & 0 & 4 & Deficient \\
\hline & June & 2 & 2 & 0 & 4 & Deficient \\
\hline & April & 1 & 1.5 & 0 & 2.5 & Very Deficient \\
\hline \multirow[t]{3}{*}{ Doctor's Office } & May & 1.5 & 2 & 0 & 3.5 & Deficient \\
\hline & June & 2 & 2 & 0 & 4 & Deficient \\
\hline & April & 2 & 1.5 & 1.5 & 5 & Deficient \\
\hline \multirow[t]{3}{*}{ Laboratory } & May & 2 & 2 & 1.5 & 5.5 & Acceptable \\
\hline & June & 2 & 2 & 1.5 & 5.5 & Acceptable \\
\hline & April & 1.5 & 1.5 & 0 & 3 & Very Deficient \\
\hline \multirow[t]{2}{*}{ Emergency } & May & 1.5 & 1.5 & 0 & 3 & Very Deficient \\
\hline & June & 1.5 & 2 & 0 & 3.5 & Deficient \\
\hline
\end{tabular}


SE

Vol. 1, $\mathrm{N}^{\circ}$ 1, 2017

\section{Journal of Sciences and Engineering}

A publication of

CINCADER

Centre of Research and Training for Regional Development Online at www.journals.cincader.org

Copyright (C) 2017, CINCADER.

ISSN 2523-9503

\section{Determination of Environmental Impacts}

Table 7. Modified Leopold Matrix of Environmental Impacts

\begin{tabular}{|c|c|c|c|c|c|c|c|c|c|c|c|c|c|c|c|}
\hline \multirow[b]{2}{*}{$\begin{array}{l}\text { Affected } \\
\text { elements }\end{array}$} & \multirow[b]{2}{*}{$\begin{array}{c}\text { Sub } \\
\text { component }\end{array}$} & \multirow[b]{2}{*}{$\begin{array}{c}\text { FACTOR } \\
\text { AMBIENTAL }\end{array}$} & \multicolumn{5}{|c|}{ Segregation } & \multirow{2}{*}{\begin{tabular}{|c|} 
Treatment \\
$\begin{array}{c}\text { Autoclaving } \\
\text { of laboratory } \\
\text { waste }\end{array}$
\end{tabular}} & \multirow{2}{*}{\begin{tabular}{|c|}
$\begin{array}{c}\text { Internal } \\
\text { transport }\end{array}$ \\
$\begin{array}{c}\text { Internal } \\
\text { Transportatio } \\
\mathrm{n} \text { of Hospital } \\
\text { Solids } \\
\text { Residues }\end{array}$ \\
\end{tabular}} & \multirow{2}{*}{\begin{tabular}{|c|}
$\begin{array}{c}\text { Cleaning and } \\
\text { disinfection }\end{array}$ \\
$\begin{array}{c}\text { Car wash for } \\
\text { internal } \\
\text { transfer }\end{array}$
\end{tabular}} & \multirow{2}{*}{\begin{tabular}{|c|}
$\begin{array}{c}\text { Central } \\
\text { storage }\end{array}$ \\
\\
$\begin{array}{c}\text { Inner time } \\
\text { storage of } \\
\text { Hospital Solid } \\
\text { Wastes }\end{array}$ \\
\end{tabular}} & \multirow{2}{*}{\begin{tabular}{|c|}
$\begin{array}{c}\text { Cleaning and } \\
\text { disinfection }\end{array}$ \\
Washing of \\
infrastructur \\
e for \\
temporary \\
collection \\
$\begin{array}{c}\text { Internal solid } \\
\text { waste }\end{array}$
\end{tabular}} & \multicolumn{2}{|c|}{ External transport } & \multirow[b]{2}{*}{ Total } \\
\hline & & & $\begin{array}{c}\text { Handling of } \\
\text { surgical } \\
\text { waste, } \\
\text { punches, etc. }\end{array}$ & $\begin{array}{c}\text { Accident risk } \\
\text { due to sharps } \\
\text { objetcs }\end{array}$ & $\begin{array}{l}\text { Handling } \\
\text { waste from } \\
\text { clinical } \\
\text { analysis }\end{array}$ & $\begin{array}{c}\text { Handling of } \\
\text { special waste }\end{array}$ & $\begin{array}{l}\text { Handling of } \\
\text { common } \\
\text { waste }\end{array}$ & & & & & & $\begin{array}{l}\text { External } \\
\text { transfer and } \\
\text { disposal of } \\
\text { waste } \\
\text { outside the } \\
\text { hospital }\end{array}$ & $\begin{array}{c}\text { Potential risk } \\
\text { of hospital } \\
\text { solid waste } \\
\text { spillage }\end{array}$ & \\
\hline \multirow{3}{*}{$\begin{array}{l}\text { Physical } \\
\text { Chemists }\end{array}$} & Soil & Soil quality & -1.2 & 0 & -1.2 & -1.2 & -1.2 & 0 & -3.2 & -3 & -1.92 & -1.2 & -1.2 & -0.5 & -15.82 \\
\hline & water & water quality & 0 & 0 & -0.9 & 0 & 0 & 0 & 0 & -5.6 & -5 & -5.6 & 0 & -0.4 & -17.5 \\
\hline & Atmosphere & air quality & 0 & 0 & 0 & 0 & 0 & 0 & -2.4 & 0 & -2 & 0 & -4.2 & 0 & -8.6 \\
\hline \multirow{4}{*}{ Cultural } & $\begin{array}{c}\text { Human } \\
\text { facilities and } \\
\text { activities }\end{array}$ & Structures & 0 & 0 & 0 & 0 & 0 & 0 & 0 & 0 & -3 & -3 & 0 & 0 & -6 \\
\hline & \multirow{2}{*}{$\begin{array}{l}\text { Cultural } \\
\text { aspects }\end{array}$} & $\begin{array}{c}\text { Health and } \\
\text { security }\end{array}$ & -2 & -1.5 & -2 & -2.5 & -2.5 & -1.2 & -2.5 & -2.5 & -3 & -2.5 & -2.5 & -3.2 & -27.9 \\
\hline & & Employment & 7 & 0 & 7 & 7 & 7 & 5.6 & 7 & 7 & 6.3 & 7 & 7 & 4.9 & 72.8 \\
\hline & $\begin{array}{l}\text { Ecological } \\
\text { Relations }\end{array}$ & $\begin{array}{l}\text { Insects, } \\
\text { desease } \\
\text { vectors }\end{array}$ & 0 & 0 & 0 & 0 & 0 & 0 & 0 & 0 & -4 & 0 & 0 & -0.9 & -4.9 \\
\hline & & Total & 3.8 & -1.5 & 2.9 & 3.3 & 3.3 & 4.4 & -1.1 & -4.1 & -12.62 & -5.3 & -0.9 & -0.1 & -15.84 \\
\hline
\end{tabular}

Table 7 refers to the environmental impacts that are generated from hospital solid waste management practices. From the segregation of common and biocontaminated waste there are slightly negative impacts on the quality of the soil and water, as it has scores between -1.2 and 0.9 , due to the fact that the waste is deposited in public spaces and gardens, as well It presents a slight direct affection to the safety and health of the operators with a score ranging from -2.5 to -1.5 since events related to punctures have occurred during the direct handling of the waste. During internal transport practices there are slightly negative impacts on the quality of soil, air and health and security of workers with scores of $-3.2,-2.4,-2.5$. During the practices of cleaning and disinfection of the equipment there is a critical point with a moderate impact (score of -5.6) towards the quality of the water since this activity is carried out frequently 2 or 3 times a day and making use of aggressive disinfectants in order to eliminate or reduce pathogens, these waters are finally poured down gutters to the drain without any previous treatment or in some cases part of these waters reach the fertile soil causing it to lose its quality. During the storage stage and the temporary collection of waste, there are slight negative impacts on the quality of soil, water and air, as well as the infrastructure and the safety and health of the workers.

The environmental factors that receive the greatest impact are the quality of water and the safety and health and security of workers with scores of -17.5 and -27.9 respectively. With respect to water quality, it is mainly impacted during the cleaning and disinfection stage and the equipment used in the management of hospital solid waste and the lack of use of personal protective equipment makes this also an environmental factor that is strongly impacted.

Within the stage of management of HSW that causes greater impact is the storage and collection of solid waste since at a given time the waste has contact with environmental components such as soil quality, water, air, obtaining a negative value of $-15.82,-17.5$ and -8.6 respectively.

However, there are also moderately positive impacts on the stages and practices of solid waste management, since these activities necessarily require an operator to execute them, promoting the generation of employment, their score ranges from 4.9 to 7 . It should be noted that it has a total value of 72.8 for the generation of employment as a single environmental factor. 
SE

Vol. 1, $\mathrm{N}^{\circ}$ 1, 2017
Journal of Sciences and

Engineering

Copyright @ 2017, CINCADER.

ISSN 2523-9503

In general, current solid waste management practices generate high negative impacts, obtaining a total value of -15.82 .

\section{Conclusions}

Due to the inadequate handling and segregation of solid waste, it generates a greater quantity of biocontaminated solid waste. On the other hand some biocontaminated waste is considered as common waste and is treated as such. Due to this inadequate practice, approximately $5 \%$ of reusable waste is contaminated and loses any opportunity to give it a new useful life.

The Hospital does not have an established route nor a collection frequency because the generation of solid waste in the different services are very variable. During the days of field verification, this situation was observed mostly in the services that are on the first floor such as Medicine I, NICU, ICU, Emergency. The waste is temporarily deposited in corridors, green areas waiting for cleaning personnel to pick them up.

Due to the cleaning and disinfection of the equipment used in the management of solid waste, the risk of proliferation of vectors, insects or rodents diminishes, although moderately negative impacts are generated for water quality, since at this stage disinfectant products with a Frequency of 3 times a day for computers and 1 time a day for storage infrastructure in order to eliminate agents.

The safety and health of workers as a single environmental factor is highly impacted because it obtains a value of -27.9. The main causes are focused on the lack of protection equipment during the stages of handling, segregation, transport or storage. There is no respiratory, visual and hand protection for $30 \%$ of the operating personnel. As a consequence, there is the risk of suffering from an occupational disease.

There is a relationship between solid waste management practices at the Alberto Sabogal Sologuren National Hospital and environmental impacts. The environmental factors that are moderately impacted are the quality of water and the safety and health of workers with an assessment of -17.5 and -27.9 respectively. However, the HSW management practices also have a positive impact on the environmental factor of employment generation, obtaining a positive value of 72.8 .

\section{Acknowledgments}

We gratefully acknowledge to University Cesar Vallejo and to the Institute of Science and Engineering, Centre of Research and Training to the Regional Development, (In Spanish, Centro de Investigacion y Capacitacion para el Desarrollo Regional-CINCADER) for allowing us to share technical experiences. 


\section{SEj}

Vol. 1, $\mathrm{N}^{\circ}$ 1, 2017
Journal of Sciences and

Engineering

Copyright @ 2017, CINCADER.

ISSN 2523-9503

\section{CINCADER}

Centre of Research and Training for

Regional Development

Online at www.journals.cincader.org

\section{Reference}

Guía para la caracterización y análisis de residuos sólidos generados en centros de salud [en línea]. [Fecha de Consulta: 03 de noviembre del 2015].

Disponible en: http://www.bvsde.paho.org/bvsacd/residuos/villena1.pdf

Ley N²7314.- Ley General de Residuos Sólidos, D.S. 057-2004-PCM. Diario oficial El Peruano, Lima, Perú, 20 de julio del 2000

Ministerio de Salud - MINSA. RM N³73-2010/MINSA - Documento Técnico Plan Nacional de Gestión de Residuos Sólidos en Establecimientos de Salud y Servicios Médicos de Apoyo 2010-2012. Lima, Perú: 2010. 46 pp.

MINSA. 2012. Ministerio de Salud. Norma Técnica de Salud № 096-MINSA/DIGESA V.01Gestión y Manejo de Residuos Sólidos en Establecimientos de Salud y Servicios Médicos de Apoyo a nivel Nacional. Lima, Perú:

http://www.minsa.gob.pe/dgsp/observatorio/documentos/infecciones/RM554-

2012_Gestion\%20y\%20Manejo\%20Residus\%20solidos.pdf

RAMÍREZ, Sandra. Formulación del plan de manejo de residuos hospitalarios en la empresa social del estado, Hospital Santo Domingo Savio del municipio del Playón Santander. Tesis (Ingeniero Ambiental). Bucaramanga, Colombia: Universidad Pontificia Bolivariana, 2009.

Disponible en: https://repository.upb.edu.co/handle/123456789/472?show=full 